\title{
The effects of initial void and dislocation on the onset of plasticity in copper single crystals
}

Cite as: J. Appl. Phys. 126, 165104 (2019); https://doi.org/10.1063/1.5125061

Submitted: 21 August 2019 . Accepted: 08 October 2019 . Published Online: 23 October 2019

Jianqiao Hu (D), Xuan Ye, Xiaoming Liu (D), and Zhen Chen (iD)
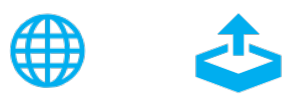

View Online

Export Citation

\section{ARTICLES YOU MAY BE INTERESTED IN}

Understanding and predicting damage and failure at grain boundaries in BCC Ta Journal of Applied Physics 126, 165902 (2019); https://doi.org/10.1063/1.5111837

Thermoelastic properties of rare-earth scandates $\mathrm{SmScO}_{3}, \mathrm{TbScO}_{3}$ and $\mathrm{DyScO}_{3}$ Journal of Applied Physics 126, 165103 (2019); https://doi.org/10.1063/1.5108584

I-V characteristics of a piezoelectric semiconductor nanofiber under local tensile/compressive stress

Journal of Applied Physics 126, 164902 (2019); https://doi.org/10.1063/1.5110876

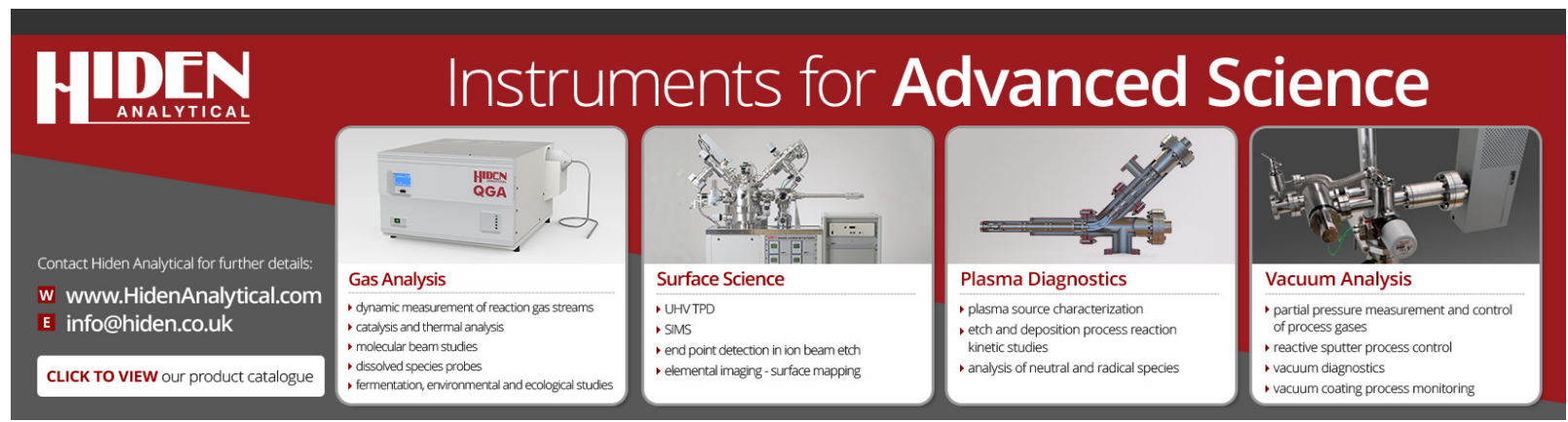




\title{
The effects of initial void and dislocation on the onset of plasticity in copper single crystals
}

\author{
Cite as: J. Appl. Phys. 126, 165104 (2019); doi: 10.1063/1.5125061 \\ Submitted: 21 August 2019 . Accepted: 8 October 2019 . \\ Published Online: 23 October 2019
}

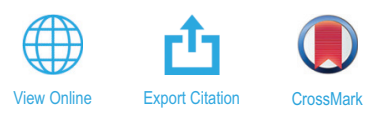

Jianqiao Hu, ${ }^{1,2}$ (D) Xuan Ye, ${ }^{7}$ Xiaoming Liu, ${ }^{1,2, a)}$ (D) and Zhen Chen $^{3,4, a)}$ (D)

\begin{abstract}
AFFILIATIONS
${ }^{1}$ State Key Laboratory of Nonlinear Mechanics, Institute of Mechanics, Chinese Academy of Sciences, Beijing 100190, People's Republic of China

${ }^{2}$ School of Engineering Science, University of Chinese Academy of Sciences, Beijing 100049, People's Republic of China

${ }^{3}$ International Research Center for Computational Mechanics, State Key Laboratory of Structural Analysis for Industrial

Equipment, Department of Engineering Mechanics, Dalian University of Technology, Dalian 116023, People's Republic of China

${ }^{4}$ Department of Civil and Environmental Engineering, University of Missouri, Columbia, Missouri 65211, USA
\end{abstract}

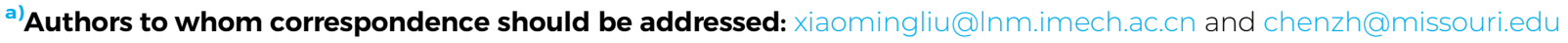

\begin{abstract}
Based on molecular dynamics, the effects of void and initial dislocation structure on incipient plasticity are investigated with single crystal copper samples. By introducing a void of different sizes and shapes and dislocations of different initial structures around the void in copper single crystals, we explore their effects on yielding as well as the dominated deformation mechanism. The main findings from this study are as follows: (1) for the samples with a void of the same size, the yield stress approaches to a specific value as the initial dislocation density increases; (2) for samples with a void of varying size, the yield stress becomes independent of the void size as it reaches a certain level, due to the saturation of preexisting dislocations around the void; and (3) as the strain rate increases, the effects of voids and preexisting dislocations on the yield stress become limited due to the response inertia, for which the phase transition from face-centered cubic to an amorphous phase occurs at an extremely high strain rate.
\end{abstract}

Published under license by AIP Publishing. https://doi.org/10.1063/1.5125061

\section{INTRODUCTION}

The initiation of ductile fracture in metallic materials is generally related to the nucleation, growth, and coalescence of voids. So far, various analytical models ${ }^{1-4}$ have been proposed to study the dynamic evolution of the void in the failure process. The emission of dislocations from the void is generally taken as the onset of plasticity and used to derive the yield strength. In particular, Lubarda et al. ${ }^{5}$ established an analytical model to evaluate the critical stress for the emission of edge dislocation from a cylindrical void and concluded that the dislocation emission plays a more crucial role than the diffusion or creep mechanism in the void growth under dynamic loading conditions. This criterion for dislocation emission is analogous to Rice and Thomson's criterion, ${ }^{6}$ which estimates the stress required for a dislocation loop emanating from a half-plane crack tip. Soon afterward, this model was debated that the emission of the shear loop has little effect on the resulting void volume. ${ }^{7}$ There are also some essential factors that are not taken into account in those analytical models such as the size effect when the void or the sample size is as small as micro-nano scale, the anisotropic nature in single crystals due to different crystalline orientation, and the strain rate sensitivity of dislocation nucleation and activity.

In addition to the analytical models, different numerical approaches have been proposed to gain further details and insight into the void-induced plasticity and the dominated deformation mechanism. Dislocation density based procedures ${ }^{8,9}$ and crystal plasticity models ${ }^{10-12}$ have been developed to investigate the void evolution and dislocation kinetics. Discrete dislocation dynamics $^{13,14}$ has also been used as an effective way to study the plastic deformation mechanism with the presence of the void. The effects of different loading situations and crystal orientations were investigated, ${ }^{15}$ with the main finding that whether the crystal orientation has an influence on the strain hardening and void growth depends on the stress states. Although a void is generally considered to be the activated location for dislocation nucleation that promotes the incipient plasticity, the obstacle strength of voids was defined by 
evaluating the critical stress for a dislocation to glide past an array of voids. ${ }^{16}$ Coincidentally, the depinning stress of an edge dislocation from a void in copper was also evaluated by the molecular dynamics (MD) simulation, ${ }^{17}$ which contains less artificial criteria or parameters in the modeling of dislocation activities. To perform a multiscale model-based simulation, the atomic-scale study is a key component for which there is a lack of understanding. With the aid of the atomistic simulation, Hatano ${ }^{18}$ studied the dislocation nucleation from a preexisting void and concluded that the Hugoniot elastic limit becomes insensitive to temperature in the presence of a void. Traiviratana et al. ${ }^{19}$ demonstrated the dislocation behaviors at different deformation stages and concluded that a larger void size would require a smaller critical stress to induce the dislocation nucleation. Zhao et al. ${ }^{20}$ studied the effects of the crystalline orientation and the void volume fraction on the incipient yield strength and macroscopic effective Young's modulus of single crystal copper. The initiation of dislocation emission was observed to account for the incipient yielding. Soon after, Tang et al. ${ }^{21}$ revealed the dislocation mechanism during the void growth and demonstrated the void size effect on the critical stress to initiate plasticity in both face-centered cubic (FCC) and body-centered cubic (BCC) metals. Temperature sensitivity, ${ }^{22}$ different stress states and strain rates, ${ }^{23}$ void geometry, ${ }^{24}$ void in nanowire, ${ }^{25}$ and array distribution of void $^{26}$ were studied to elucidate the void related plastic deformation or failure mechanism. However, most of the above studies are based on the assumption that there are no other defects around the preexisting voids initially. As a result, the emission of dislocation from the void has been considered to be the dominated mechanism accounting for the incipient plasticity. Nevertheless, it has been clarified that the preexisting dislocation has a significant effect on the onset of plasticity, e.g., the pop-in phenomenon during nanoindentation ${ }^{27}$ and the strength and the tension-compression asymmetry of nanolaminate composites. ${ }^{28}$ It has also been reported that the preexisting dislocation has a significant influence on the formation of new dislocation loops, ${ }^{29}$ which will further affect the plastic behavior of the materials. For the metallic materials that have suffered from prestrained deformation or even subjected to shock loading, voids as well as the surrounding dislocations have been observed in the metals (see Fig. 1 in Ref. 30 and Fig. 2 in Ref. 19), but they would continue to serve in many cases. Hence, an important issue arises, namely, how to evaluate the incipient yielding of the materials with both voids and surrounding preexisting dislocations?

Motivated by the above issue, the incipient yielding in copper single crystals in the presence of both void and dislocations is investigated based on molecular dynamics (MD). The remaining of the paper is organized as follows. The MD methodology and model description are presented in Sec. II. The numerical results including the effects of the void size and shape, preexisting dislocation, and loading rate on the incipient yielding stress are demonstrated and analyzed in Sec. III. Finally, concluding remarks are summarized in Sec. IV.

\section{METHODOLOGY AND MODEL DESCRIPTION}

The large-scale atomic/molecular massively parallel simulator ${ }^{31}$ is employed in our MD simulations. The geometry of copper single crystals containing void is illustrated in Fig. 1(a), which only shows half of the atoms to illustrate the void in the center. A cubic box cell full of copper atoms with the FCC structure is established first; then a void of different size and shape is generated in the center of the box cell, which is also the origin of the coordinate axis. In the atomic model, the $x, y$, and $z$-axes are oriented in the [100], [010], and [001] lattice directions, respectively. The size of box cell is $60 a \times 60 a \times 150 a$ with $a$ being the lattice constant of $3.615 \AA$. The diameter of the spherical void at the center of box cell ranges from $6 a$ to $40 a$, while the void is also set to cubes with side lengths from $6 a$ to $40 a$ to study the shape effect (Fig. 1 only shows the spherical void). Periodic boundary conditions are employed along all the three sides to mimic the bulk behavior. The interaction among copper atoms is described by the embedded atom method (EAM) poten$\mathrm{tial}^{32}$ which has been demonstrated accurately in the description of both the unstable and stable stacking fault energies. ${ }^{33}$ The Mishin potential has been widely used in the simulation of copper including the shock compression, ${ }^{3-36}$ the hydrostatic pressure effects, ${ }^{37}$ the homogeneous dislocation nucleation, ${ }^{38,39}$ and hydrostatic and shockinduced melting. ${ }^{40}$

Starting from the initial atomic configuration, the atomic model was firstly equilibrated in the isobaric-isothermal (NPT) ensemble at a pressure of $0 \mathrm{GPa}$ and temperature of $300 \mathrm{~K}$ for $10 \mathrm{ps}$. Then as shown in Fig. 1(b), with the same temperature $300 \mathrm{~K}$, the atomic configuration was loaded with an NPT ensemble under a hydrostatic pressure state, which linearly increases from $0 \mathrm{GPa}$ to a specific pressure $P_{0}$ and further linearly releases to $0 \mathrm{GPa}$ with a constant rate of $0.5 \mathrm{GPa} / \mathrm{ps}$. Subsequently, an initial minimization of the sample was performed for $10 \mathrm{ps}$ with an NPT ensemble at $0 \mathrm{GPa}$ and $300 \mathrm{~K}$ before proceeding uniaxial tension. Through this preloading and unloading process, as shown in Fig. 1(c), different dislocation structures are generated around the void, depending on the preloaded hydrostatic pressure, void size, and shape. Based on the above atomic configurations, uniaxial tension loading at a constant strain rate of $1 \times 10^{9} \mathrm{~s}^{-1}$ was applied on the $z$-direction and the global pressure of the sample at the other two lateral directions (i.e., $x$ and $y$ directions) was kept at $0 \mathrm{GPa}$. This process was accomplished by decoupling the strained dimension for NPT in the two lateral directions, ${ }^{41,42}$ and the temperature was kept at $300 \mathrm{~K}$. To reveal the strain rate effect, (a)

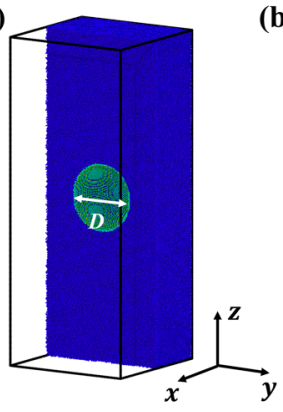

(b)

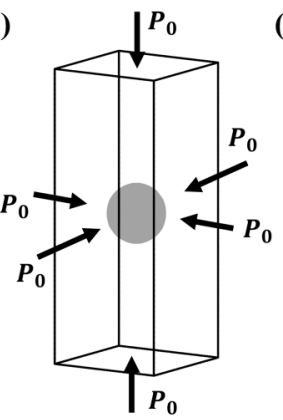

(c)

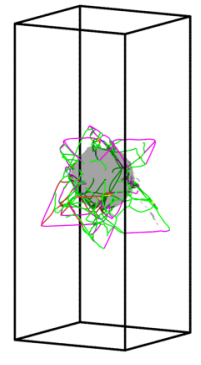

FIG. 1. The schematic diagram of copper single crystals containing void and dislocations. (a) Void in copper single crystals, (b) hydrostatic loading and unloading processes, and (c) initial dislocations generated around the void. 
different strain rates that varied from $5 \times 10^{7} \mathrm{~s}^{-1}$ to $1 \times 10^{11} \mathrm{~s}^{-1}$ were also carried out. The time step is set to $1 \mathrm{fs}$, except for the strain rate of $1 \times 10^{11} \mathrm{~s}^{-1}$, a shorter time step of $0.1 \mathrm{fs}$ is used. The tension loading continues until the maximal strain reaches about $15 \%$ when the strain rate is below $5 \times 10^{9} \mathrm{~s}^{-1}$; otherwise, the maximal strain is $\sim 30 \%$. The overall system stress is calculated as the volumetric average of the local stress on each atom derived from the virial theorem, ${ }^{43}$ as used in previous MD simulations. ${ }^{44,45}$ In the analysis, tracking of dislocations is achieved by the dislocation extraction algorithm (DXA), ${ }^{46}$ and Ovito ${ }^{47}$ is utilized to visualize the defect structures. The yield stress in the analysis is defined as stress value corresponding to the incipient stress drop as the uniaxial tension continues. The dislocation density is calculated by dividing the total length of the dislocation line by the volume of the box cell.

\section{RESULTS AND ANALYSIS}

\section{A. Samples with void and initial dislocation}

In this subsection, the effect of the void size as well as preexisting dislocations on the mechanical behavior of copper single crystals is studied. First, the preexisting dislocations under different hydrostatic pressure $P_{0}$ are studied in samples with void of two distinct sizes as shown in Fig. 2(a), two features can be obtained that the dislocation density $\rho_{0}$ becomes saturated and stable in both cases as the hydrostatic pressure exceeds a critical value; there is a dramatic increase in the saturated dislocation density (from $\sim 0.02 \mathrm{~nm}^{-2}$ to $0.18 \mathrm{~nm}^{-2}$ ) when the void diameter changes from $12 a$ to $30 a$. The dislocation structures under some specific pressure values are illustrated as shown in Fig. 2(b). The type of dislocation is distinguished by different colors, the Shockley partial dislocations and stair-rod dislocations are particularly indicated since they are far more numerous than other types. Since the preexisting dislocations have an effect on the mechanical behavior of the subsequent uniaxial tensile loading, the atomic deformation map is presented to interpret the evolution of dislocation under hydrostatic loading as shown in Fig. 3, the atoms are colored by the level of their centrosymmetry parameters (CSP). It can be seen clearly the void has collapsed under the high hydrostatic pressure that sustains the final saturated dislocation density. The dislocations nucleate from the surface of the voids with the aid of an external load. The emission and motion of dislocations rearrange the atoms around the void. With the increase of hydrostatic pressure, these dislocation activities result in the shrinkage and collapse of the void, and then the dislocations reach saturated. The large void can provide more space to generate dislocations such that the dislocations would spread widely, while the surrounding dislocations are trapped in a limited space around the small void.

Next, based on these samples with a void of different size and initial dislocation structures, uniaxial tension at a constant strain rate of $1 \times 10^{9} \mathrm{~s}^{-1}$ was performed. The strain-stress curves as well as the evolution of dislocation densities are presented in Fig. 4. It can be seen from Fig. 4(a) that for samples with a relatively small void size (i.e., $D=12 a$ ), the stress first increases linearly, then followed a sharp decrease in the stress level with the increase of strain. Corresponding to the evolution of stress, the dislocation density is stable at first and then increases drastically as shown in Fig. 4(b). The dislocation density keeps almost constant at the initial deformation stage because the dislocations are jammed in a limited space around the void and the stress is not high enough to motivate these dislocations. It has been analyzed above that the generated initial dislocation density would increase to a convergent value with the increase of $P_{0}$. As a result, there is almost no difference in the corresponding stress-strain curves when $P_{0}$ exceeds $16 \mathrm{GPa}$ and the yield stress also becomes stable (approximately $3.5 \mathrm{GPa}$ ). Similarly, for samples with the larger void of diameter $30 a$ as shown in Figs. 4(c) and 4(d), the effect of $P_{0}$ on the initial dislocation density is similar to that in samples with small voids despite that the initial dislocation density exhibits some differences when $P_{0}$ is larger than $11 \mathrm{GPa}$. The peak stresses in the stress-strain curves also converge to a stable value of $\sim 2.3 \mathrm{GPa}$ when $P_{0}$ exceeds $11 \mathrm{GPa}$. It should be pointed out that the initial dislocation density is slightly different from that in Fig. 2(a) since there is a relaxation (a)

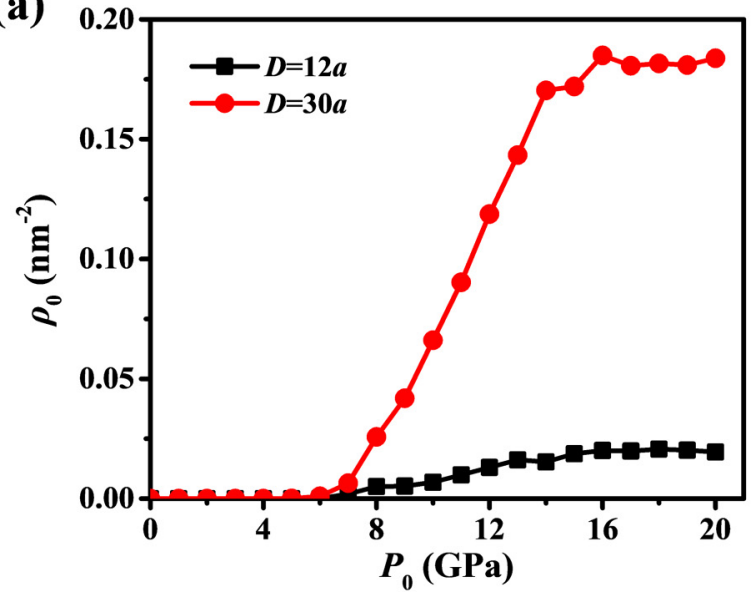

(b)
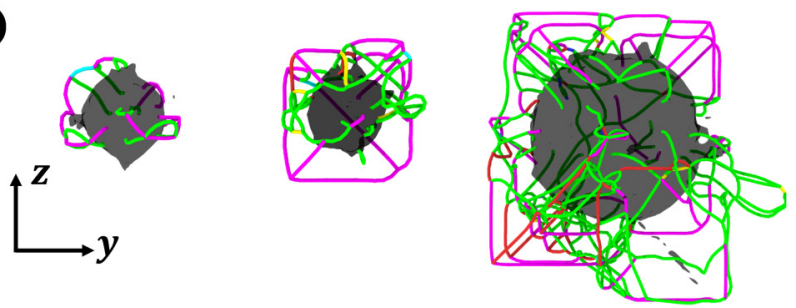

$$
\begin{array}{ccc}
D=12 a & D=12 a & D=30 a \\
P_{0}=12 \mathrm{GPa} & P_{0}=14 \mathrm{GPa} & P_{0}=8 \mathrm{GPa}
\end{array}
$$

$1 / 6<112>$ Shockley partial dislocation

$1 / 6<110>$ Stair-rod dislocation

FIG. 2. (a) The dislocation density under different hydrostatic pressure $P_{0}$ and (b) the different initial dislocation structures around the spherical void of different sizes. 


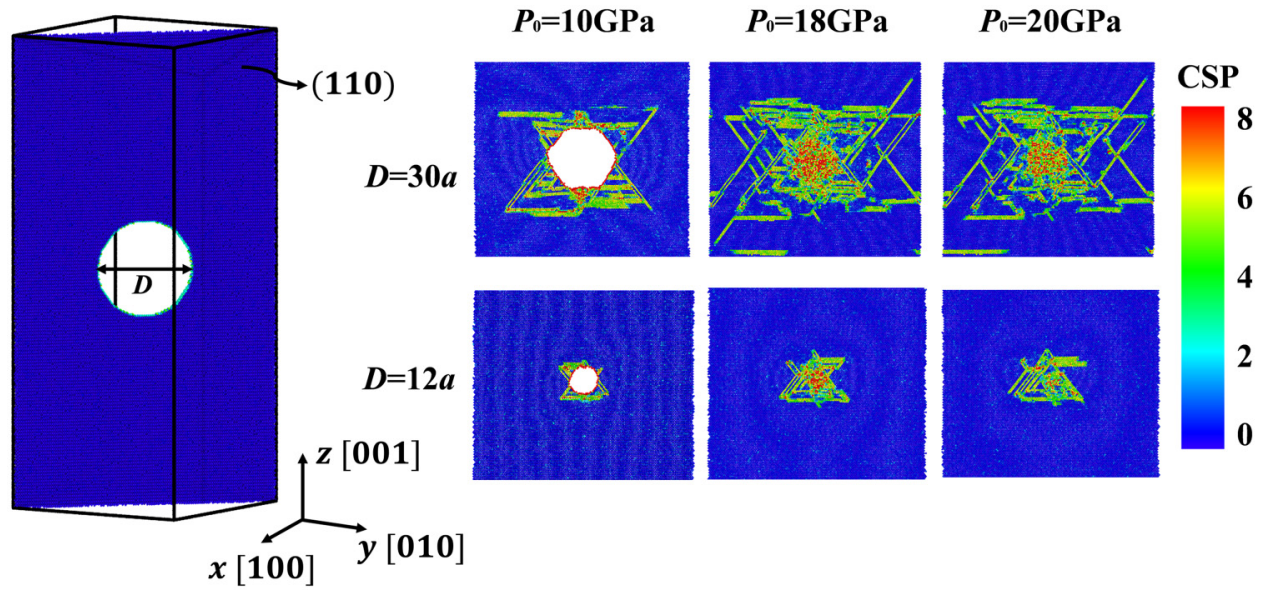

FIG. 3. The CSP of atoms around the void of different sizes at the view of plane (110). process when the dislocations would evolve dynamically before tensile loading. The evolution of dislocation density also exhibits some new features in Fig. 4(d), the dislocation density would first decrease slightly, and it indicates that dislocation would interact with each other and annihilate at this stage. Corresponding to the decrease of stress in Fig. 4(c), the dislocation density would increase slowly again and then become stable. Comparing Figs. 4(a) and 4(c), the yield stress exhibits different sensitivities to the hydrostatic pressure $P_{0}$. It is because the amount of dislocation density is quite limited in the sample with small void $(D=12 a)$, the yield stress is relevant to these preexisting dislocations. While for samples with void of $D=30 a$ at a relatively low hydrostatic pressure, the dislocation density is already larger than the saturated density around the small void, and then the yield stress exhibits less sensitivity to the initial dislocations. In addition, for samples with equivalent amount of dislocations and void of different sizes [see the dark cyan line in Fig. 4(b) and the red line in Fig. 4(d)], the dislocation densities are $\sim 0.025 \mathrm{~nm}^{-2}$ initially but evolve in a (a)

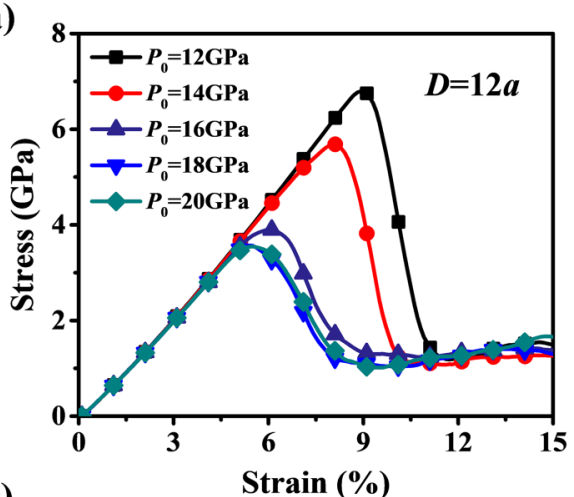

(c)

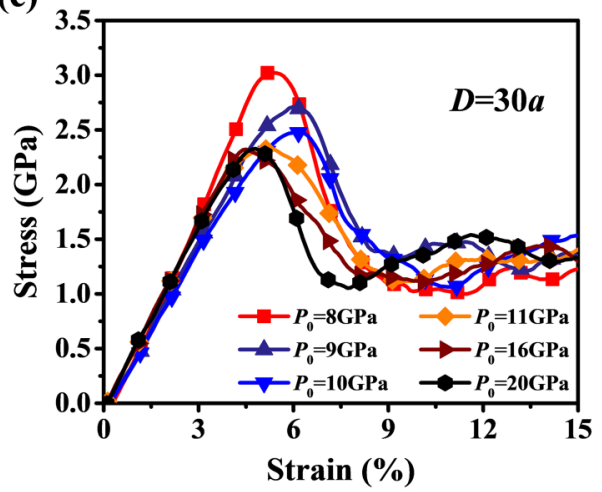

(b)

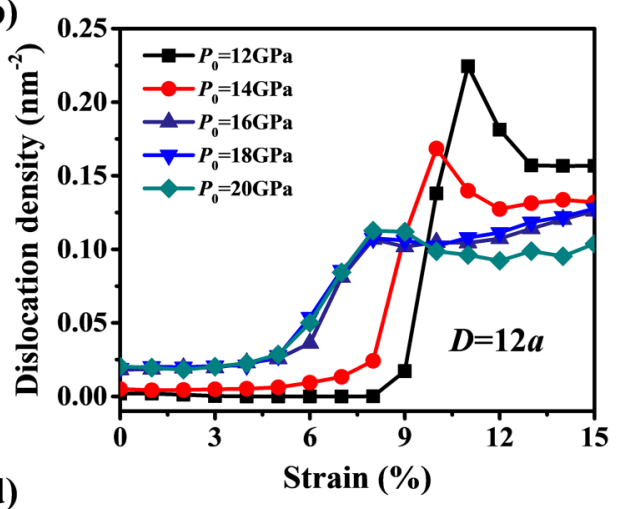

(d)

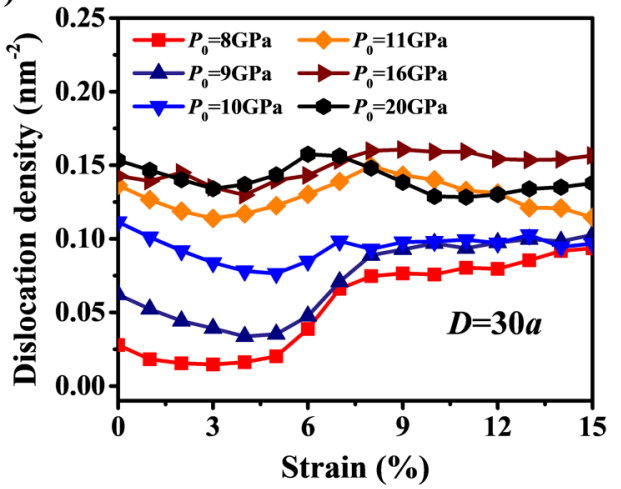

FIG. 4. The stress-strain and the dislocation density-strain curves corresponding to samples consisting of void [(a) and (b)] $D=12 \mathrm{a}$ and [(c) and (d)] $D=30$ a at a constant strain rate of $1 \times 10^{9} \mathrm{~s}^{-1}$. 
slightly different way with the increase of strain, and the incipient yield stress is slightly smaller in the sample with large void of $D=30 a(\sim 3.0 \mathrm{GPa})$ than that $(\sim 3.5 \mathrm{GPa})$ of the sample with void of $D=12 a$.

Through the above analysis, when the initial hydrostatic pressure exceeds a specific value during sample preparation, there is almost no change in the initial dislocation density and the yield stress during uniaxial tension decreases to a stable value. It is worth noting that the starting hydrostatic pressure that saturates the dislocations is related to the void size. Despite this difference, it is confirmed that the hydrostatic pressure $20 \mathrm{GPa}$ can make the dislocations around the void saturated in the range of void sizes we studied. Next, based on this conclusion, the yield stress is further investigated in samples fabricated by the hydrostatic pressure $20 \mathrm{GPa}$.

\section{B. Effect of void size}

In this subsection, the effect of the void size on the yield stress at the strain rate of $1 \times 10^{9} \mathrm{~s}^{-1}$ is studied in samples with saturated initial dislocation density that corresponds to the hydrostatic pressure $20 \mathrm{GPa}$. As shown in Fig. 5(a), the samples with void of different sizes generally yield at different strains and the yield stresses exhibit a void size dependence when the void size is less than $18 a$. It can also be seen that there is almost no change in the peak stress (i.e., the yield stress) when the void size increases to a certain extent. Correspondingly, the evolution of dislocation density also varies depending on the void size. For samples with a larger void, the dislocation density will slightly decrease at first and then increases and, finally, evolves to a stable state, while it will dynamically increase to a stable value when the void size is small. Furthermore, when the void size reaches $30 a$, which is comparable to the lateral size of the box cell, the removal of material in the void affects the elastic modulus [i.e., the slope of the curves at the initial deformation stage in Fig. 5(a)].

Similar to the yielding process during a dynamic loading, ${ }^{48}$ two distinct dislocation activities can be proposed to explain the void size-related yield stress, the plastic shear strain rate can be written as: ${ }^{49} \dot{\gamma}=b \rho \bar{v}+b \dot{\rho} \bar{x}$, where $b$ is the magnitude of the Burgers vector, $\rho$ is the dislocation density, $\bar{v}$ is the average dislocation velocity, and $\bar{x}$ is a displacement during the formation of the generated dislocations. This formula naturally introduces two different dislocation behaviors during the plastic deformation, the first item presents the motion of preexisting dislocations and the second one indicates the generation of new dislocations. The dominated dislocation behavior can be obtained by analyzing the evolution of dislocation density in Fig. 5(b) as well as the dislocation structures illustrated in Fig. 6. For samples with relatively small void (e.g., $D=6 a$ and $12 a$ ), the amount of preexisting dislocations, which depends on the void size, is quite limited such that the generation of new dislocations is required. It can be seen from Fig. 6(a) that there has been an apparent dislocation multiplication before yielding and the increase of dislocation density when yielding occurs is contributed by the collaboration of dislocation nucleation from the preexisting dislocation network and dislocation multiplication away from the central area. In this case, the second item dominates the yielding, characterized by the apparent increase in dislocation density [see Fig. 5(b)]. As a result, the yield stress exhibits a void size dependence due to the void size-related initial dislocations. In contrast, for the sample with a void of diameter $D=30 a$ as shown in Fig. 6(b), dislocations jammed in complex structures and maintained quite stable before yielding. The motion of these preexisting dislocations is sufficient to generate enough plasticity, in which the change of dislocation density is moderate and the first item is dominated. The yield stress shows the independence of the void size. Moreover, for samples in the deformation stage of the stable plastic flow (i.e., $\varepsilon>\sim 11 \%$ ) as shown in Fig. 5(a), the flow stress sustains at approximately $1.2 \mathrm{GPa}$ and the corresponding dislocation density is $\sim 0.1 \mathrm{~nm}^{-2}$. Coincidentally, the yield stress and the evolution of dislocations generally exhibit two different characteristics in samples whose initial dislocation density is higher or less than $\sim 0.1 \mathrm{~nm}^{-2}$. The dislocations start to show some statistical characteristics when they are sufficient, and the stress to motivate these dislocations remains relatively constant. Since the transition from void size-dependence to independence is correlated to the initial dislocation density, it can be referred there would be a critical void size achieving this transition.

Furthermore, the yield stresses above as well as those for samples with spherical void but without initial dislocation (i.e., when $P_{0}=0 \mathrm{GPa}$ ) are presented in Fig. 7. For samples without preexisting dislocations, the yield stress exhibits a conventional void (a)

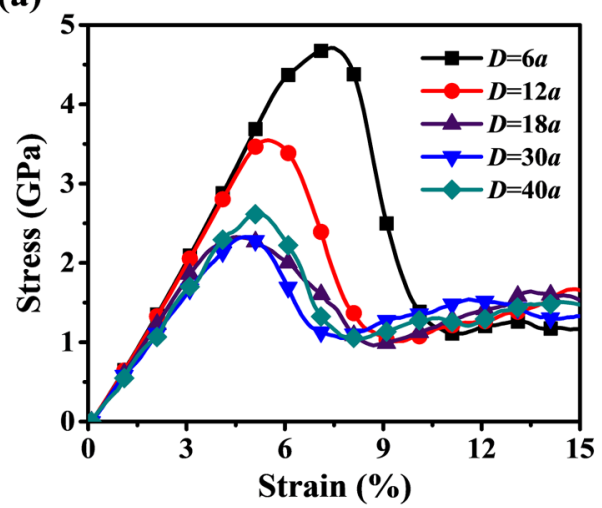

(b)

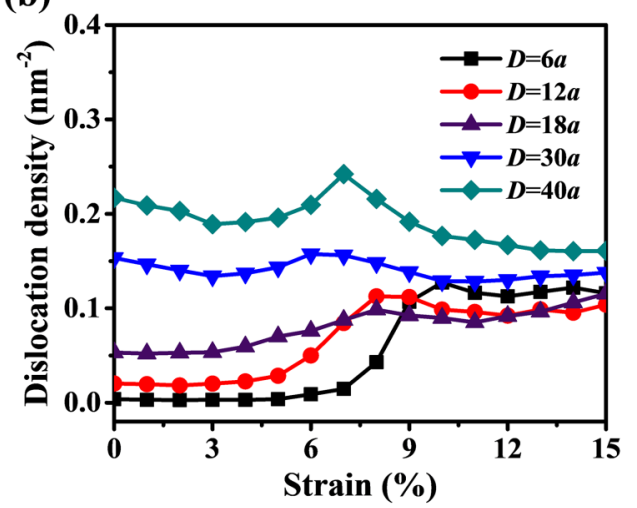

FIG. 5. (a) The stress-strain curves and (b) the dislocation density-strain curves corresponding to samples with void of different sizes at a constant strain rate of $1 \times 10^{9} \mathrm{~s}^{-1}$. 
(a)

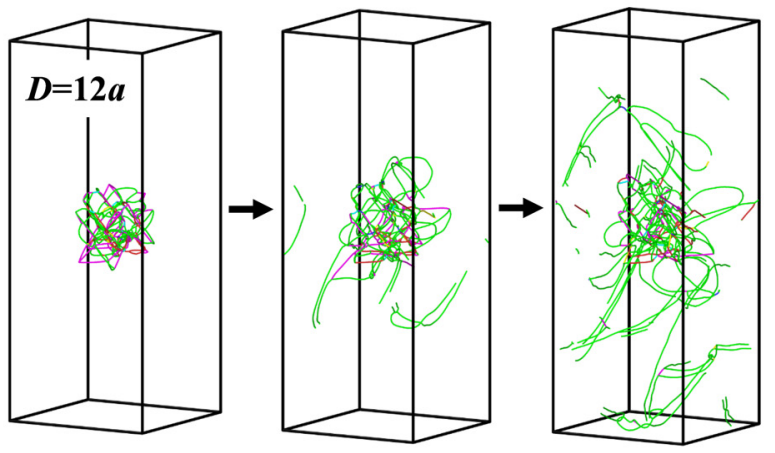

(b)

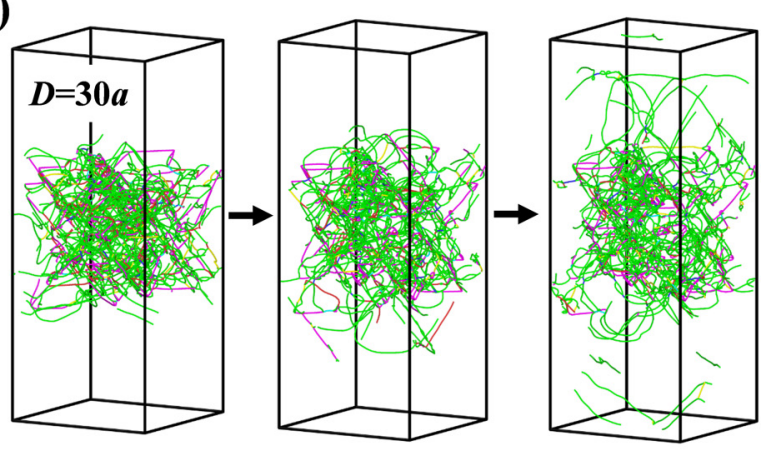

FIG. 6. The dislocation structures at initial, before and when yielding occurs in samples initialized under $P_{0}=20 \mathrm{GPa}$ with void of diameter (a) $D=12 \mathrm{a}$ and (b) $D=30 \mathrm{a}$.

size effect, that is, a larger void results in smaller yield stress. This void size effect without preexisting dislocations has been thoroughly discussed that the critical stress for the emission of dislocation from the void surface is size-related. ${ }^{5,30}$ However, it can be seen from Fig. 7 that the existence of initial dislocation has two profound effects on the yield stress. First, there is a significant drop in the value of yield stress due to the presence of initial dislocations. It demonstrates that the critical stress to activate the preexisting dislocations is much smaller than that to trigger the dislocation nucleation from the surface of the void. Second, the yield stress becomes independent of the void size as the void size increases. This trend also applies to the sample with a cubic void, exhibiting a void shape independence. However, in the sample with cubic void, the critical size when the yield stress starts to exhibit the independence of the void size is smaller $(D=12 a)$. Correspondingly, the preexisting saturated dislocation density is $0.025 \mathrm{~nm}^{-2}(D=12 a)$, which is larger than that $\rho_{0}=0.02 \mathrm{~nm}^{-2}$ in samples with spherical void. From the above analysis, when there are enough preexisting

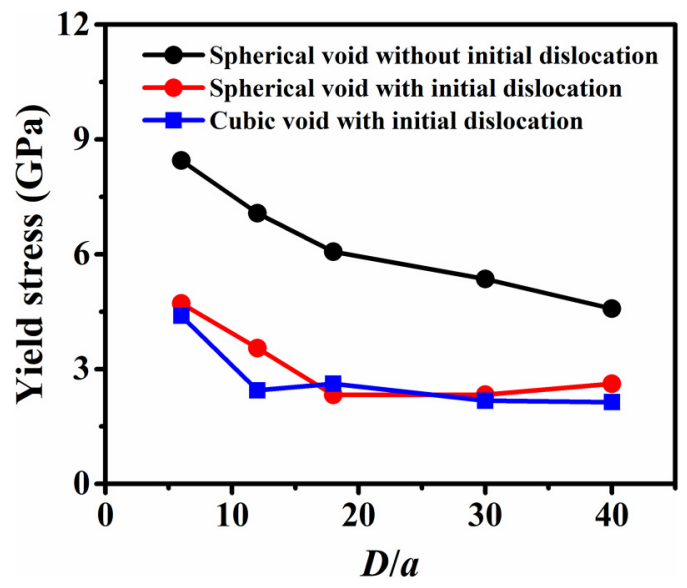

FIG. 7. The yield stress for the sample with void of different sizes and shape at a constant strain rate of $1 \times 10^{9} \mathrm{~s}^{-1}$. dislocations surrounding the void, it is the activation of these dislocations that leads to yielding, which is insensitive to the void size and different from dislocation nucleation mechanism in the absence of initial dislocations.

\section{Effect of loading rate}

The effect of void and preexisting dislocations has been studied at a certain strain rate. In this subsection, different loading rates are further performed to revisit the plastic behavior above. Strain rates ranging from $5 \times 10^{7} \mathrm{~s}^{-1}$ to $1 \times 10^{11} \mathrm{~s}^{-1}$ are applied on samples with void of two different sizes in which the preexisting dislocations are generated by the hydrostatic pressure of $P_{0}=20 \mathrm{GPa}$.

As shown in Fig. 8, the stress-strain curves as well as the evolution of dislocations is studied for samples with void of two different sizes under different strain rates. From Figs. 8(a) and 8(c), samples generally exhibit the conventional strain rate effect, namely, larger peak stress under a higher strain rate. Coincidentally, when the strain rate is below $1 \times 10^{9} \mathrm{~s}^{-1}$, the stress-strain curves become serrated when massive yield takes place, characterized by several fluctuations in the stress and consistent with the previous MD calculations. ${ }^{20,50}$ Correspondingly, as shown in Figs. 8(b) and 8(d), the variation in dislocation density is small. For relatively low strain rate loading, the initial activation of preexisting dislocation would sustain enough plasticity for yielding. By further analyzing the dislocation structures, it was found that a number of stair-rod dislocations form inside the sample, and these immovable stair-rod dislocations serve as the obstacles for dislocation motion. ${ }^{51}$ The serrated fluctuations in the stress are caused by the pinning and depinning of dislocations. While for extremely higher strain rate exceeding $5 \times 10^{9} \mathrm{~s}^{-1}$, there is a dramatic increase in the dislocation density when the incipient yielding occurs, exhibiting the characteristics of dislocation nucleation.

The yield stresses for samples in Fig. 8 as well as for the samples with void but in the absence of initial dislocations and the perfect copper single crystals are summarized in Fig. 9(a). For all samples at a strain rate below $\sim 1 \times 10^{8} \mathrm{~s}^{-1}$, the yield stress shows rate independence. A dramatic change in the strain rate sensitivity is observed when the strain rate exceeds $1 \times 10^{9} \mathrm{~s}^{-1}$, which is consistent with the experiment measurement. ${ }^{52-54}$ Similar transformation in strain rate sensitivity is also observed in the perfect copper 

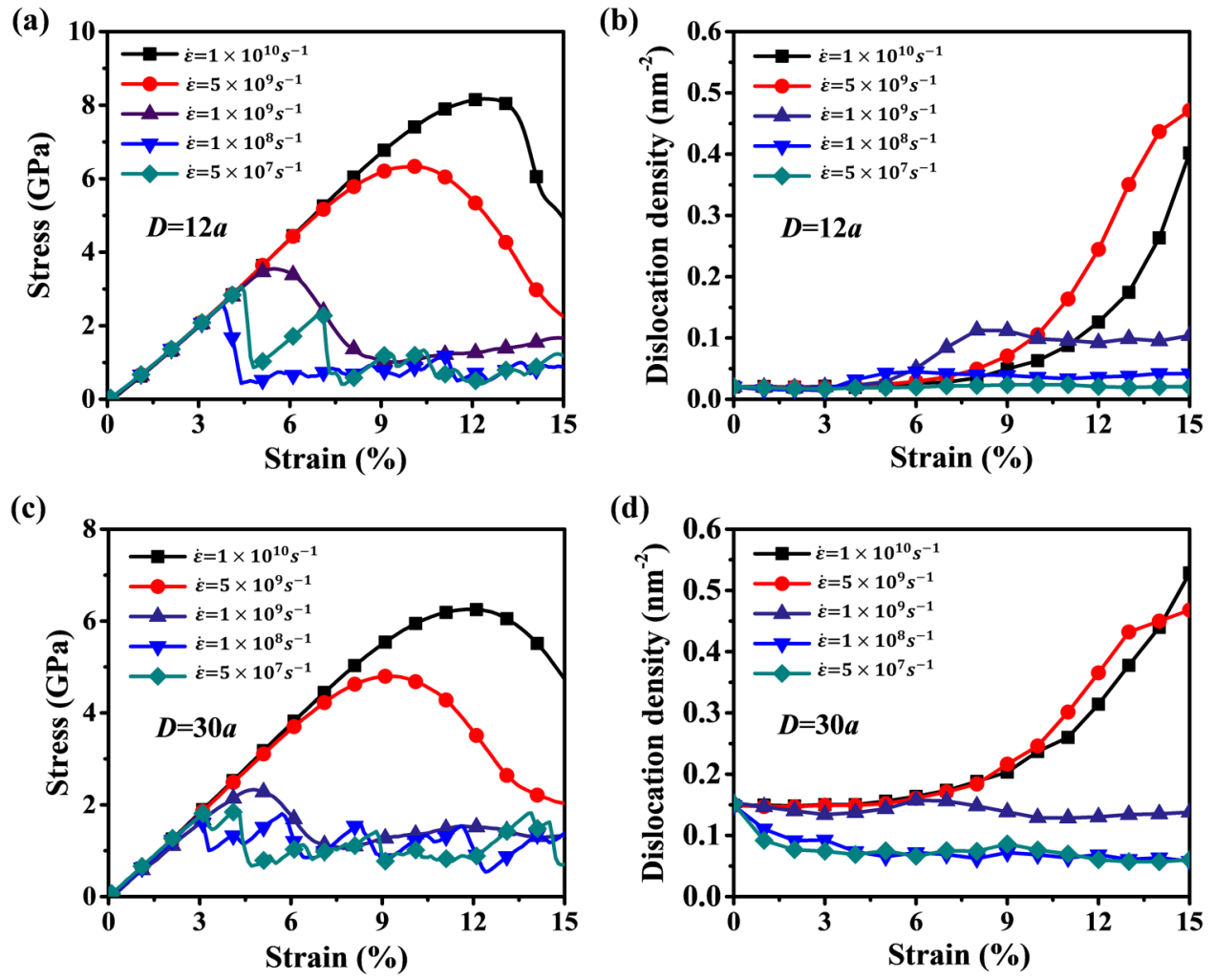

FIG. 8. The stress-strain curves and the dislocation density-strain curves for samples under different strain rates: [(a) and (b)] $D=12 a$ and [(c) and (d)] $D=30$ a. (a)

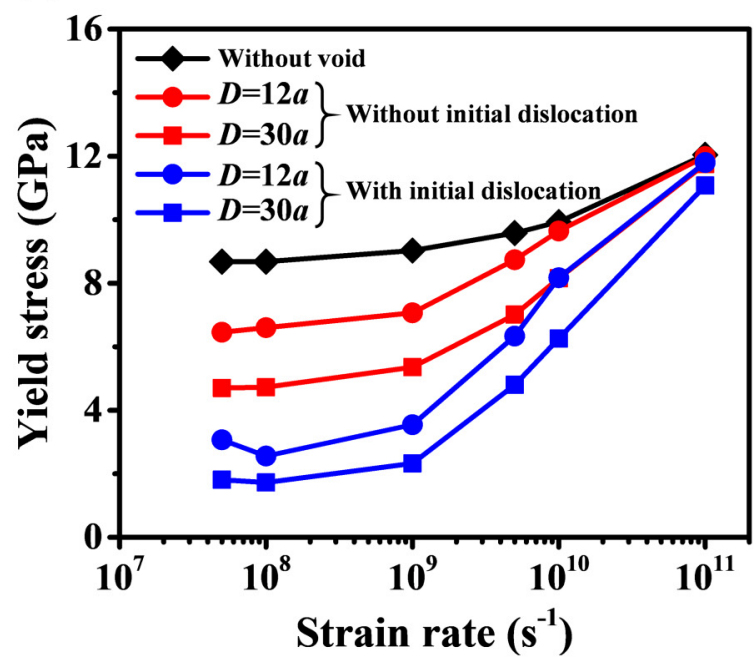

(b)

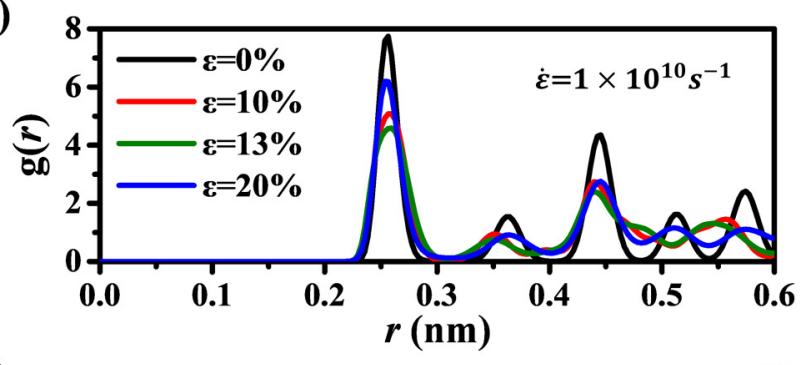

(c)
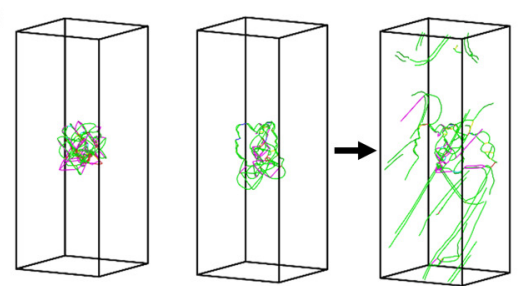

Initial dislocation $\dot{\varepsilon}=1 \times 10^{8} \mathrm{~s}^{-1}$

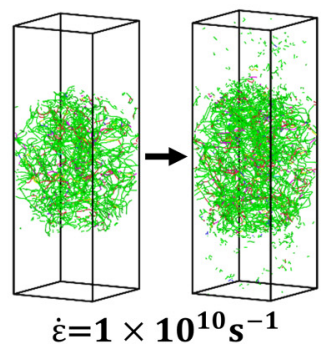

FIG. 9. (a) The yield stress of samples with and without voids and preexisting dislocations, (b) RDF of the sample in the presence of both void $D=12 a$ and dislocations at different strains, and $(c)$ the dislocation structures in samples in the presence of both void $D=12 a$ and dislocations when yielding occurs. 


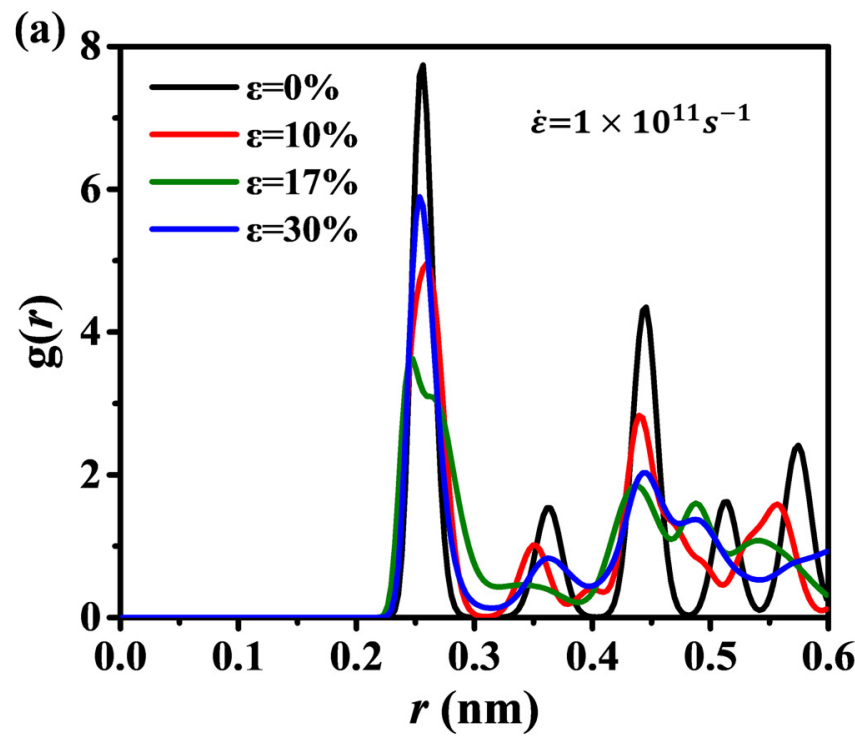

(b)

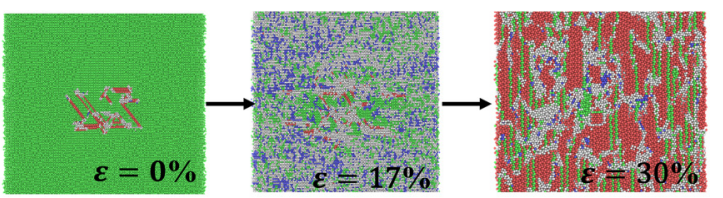

(c)

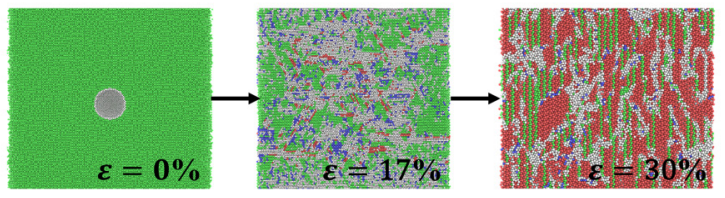

(d)
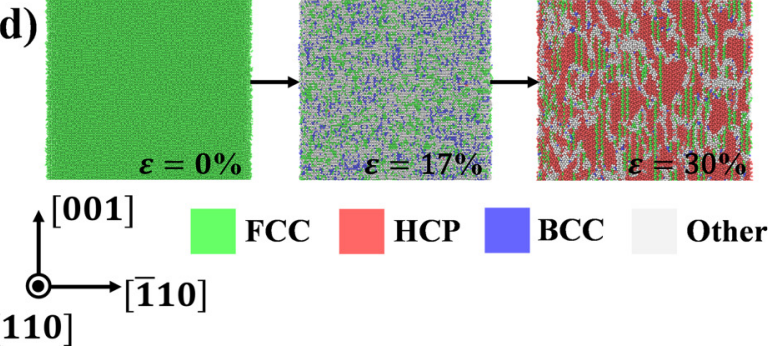

FIG. 10. (a) RDF of the sample in the presence of both void $D=12 a$ and intial dislocations. The atomic structures in samples at different strains: (b) $D=12 a$ and initial dislocations, (c) $D=12 a$ and no initial dislocations, and (d) perfect crystal with no initial defect.

single crystals in which the incipient plasticity is always considered to be related to the homogeneous nucleation of dislocation. To analyze the underlying deformation mechanism at different strain rates, as shown in Fig. 9(b), the radial distribution function (RDF), i.e., $\mathrm{g}(r)$, is studied first to confirm whether it is still the dislocation activity dominating the yielding at the strain rate $1 \times 10^{10} \mathrm{~s}^{-1}$ since it has been reported ${ }^{55}$ that phase transition would occur with the increase of strain rate. The green line with a strain of $13 \%$ corresponds the time when yielding occurs in the sample in the presence of both void $D=12 a$ and dislocations. The second nearest neighbor peak in $\mathrm{g}(r)$ around $0.36 \mathrm{~nm}$ in Fig. 9(b) clearly demonstrates that a crystalline FCC system remains at the strain up to $20 \%$ when massive plasticity gets involved. In addition, the dislocation structures in the sample with void $(D=12 a)$ and initial dislocations are illustrated in Fig. 9(c), corresponding to the time before and when yielding occurs, respectively. The initial dislocation structure is also shown in the leftmost. A significant difference in the evolution of dislocation structure can be observed under different strain rates and the dominated deformation mechanism can be divided into two regimes. For strain rate of $1 \times 10^{8} \mathrm{~s}^{-1}$, it is the activation of the preexisting dislocation dominating the yielding process since the dislocation arms are original from the dislocations surrounding the void. While for samples under the high strain rate of $1 \times 10^{10} \mathrm{~s}^{-1}$, dislocation multiplication cannot make yielding happen because there has been a significant increase in the number of dislocations around the void compared to the initial dislocation structure. In addition to the dislocation multiplication, dislocation debris away from the void is also observed in the sample when yielding takes place. It demonstrates that the dislocation nucleation significantly contributes to yield at high strain rates.

Despite that the yield stresses for samples with initial defects are generally lower than that in perfect crystal, when the strain rate increases to an extreme level, i.e., $1 \times 10^{11} \mathrm{~s}^{-1}$, as shown in Fig. 9(a), there is no distinct difference in the yield stress. The yielding process exhibits the independence of the initial defects. The RDF at this strain rate is further studied to get insight into the possible change of the atomic structures. As shown in Fig. 10(a), the $\mathrm{RDF}$ of the sample in the presence of both void $D=12 a$ and initial dislocations is given at different strains. The crystalline FCC structure can be clearly evident from the RDF in Fig. 10(a) at a strain of $0 \%$ and up to $10 \%$. However, when the yielding occurs at the strain of $17 \%$, the second peak located at around $0.36 \mathrm{~nm}$ disappears, which suggests that the crystal structure transforms into an amorphous state. To further reveal the structure transition from the FCC phase to the amorphous phase, the snapshots of the atomic structures in different samples at varying strains are displayed in Figs. 10(b)-10(d). The atomic structures in the middle column at the strain of $\sim 17 \%$ correspond to the yielding in all samples. As the strain rate increases to $1 \times 10^{11} \mathrm{~s}^{-1}$, samples deform in a similar way despite the difference in the initial defects. Similar to the high strain rate-induced amorphous phase observed in the MD simulations of single crystal nickel nanowires, ${ }^{56}$ and platinum and gold nanorods, ${ }^{57}$ the phase transition has occurred in all samples since most of the atoms initially in FCC structures change to the amorphous structures (when yielding occurs). Finally, most of the atoms are rearranged in a hexagonal close-packed (HCP) structure when 
massive plasticity takes place $(\epsilon=30 \%)$. This transition to the HCP structure has also been observed in the copper nanowire at a high strain rate, ${ }^{58}$ in which it was confirmed that the average atom potential energy in the HCP structure is located at the local minima of the energy-strain curve. The initial defect-independent yielding is own to the phase transition at an extremely high strain rate.

\section{CONCLUDING REMARKS}

Metal ductility and damage localization are generally related to the kinetics of void growth and the evolution of dislocations. For the metallic materials containing only voids, incipient plasticity is generally considered to be due to dislocation nucleation around the void, which has been quantitatively evaluated by various analytical models and demonstrated for the void size dependency. However, for metals that have been subjected to the deformation or impact, initial dislocations would exist inside the metallic material and affect the subsequent deformation process.

Based on molecular dynamics, copper single crystals with both void and preexisting dislocation subjected to uniaxial tensile loading are examined in this paper with different loading rates. The yield stress as well as the plastic deformation mechanism is studied by varying the void size and shape and the strain rates. It can be concluded from the analysis that, for the samples without preexisting dislocation, the yield stress exhibits the traditional void size dependency. For the samples containing both initial void and dislocation, however, two salient features of the yield stress can be obtained as follows: (1) the yield stress decreases to a stable value with the increase of initial dislocation density when the void size is fixed and (2) the yield stress is insensitive to the void size and shape when the void exceeds a specific size. Furthermore, with the increase of strain rate, the yield stress increases to a convergent value, regardless of the presence of initial void and dislocation inside the specimen. The dominated mechanism of incipient plasticity transforms from the nucleation/multiplication of dislocations to the phase transition (from the FCC to an amorphous state).

\section{ACKNOWLEDGMENTS}

This work is supported by the National Natural Science Foundation of China (NNSFC, No. 11802310) and the Science Challenge Project (No. TZ2018001). The work of X.L. is supported by the National Natural Science Foundation of China (Nos. 11772334 and 11672301), by the Youth Innovation Promotion Association CAS (No. 2018022), and by the Strategic Priority Research Program of the Chinese Academy of Sciences (No. XDB22040501).

\section{REFERENCES}

${ }^{1}$ A. L. Gurson, "Continuum theory of ductile rupture by void nucleation and growth: Part I-Yield criteria and flow rules for porous ductile media," J. Eng. Mater. Technol. 99(1), 2-15 (1977).

${ }^{2}$ C. L. Hom and R. M. McMeeking, "Void growth in elastic-plastic materials," J. Appl. Mech. 56(2), 309-317 (1989).

${ }^{3}$ B. Liu, X. Qiu, Y. Huang, K. C. Hwang, M. Li, and C. Liu, "The size effect on void growth in ductile materials," J. Mech. Phys. Solids 51(7), 1171-1187 (2003).
${ }^{4}$ L. Wang, J. Zhou, D. Hui, and S. Zhang, "Micromechanics model for nanovoid growth and coalescence by dislocation emission: Loading and lattice orientation effects," Int. J. Mech. Sci. 79, 168-175 (2014).

${ }^{5}$ V. A. Lubarda, M. S. Schneider, D. H. Kalantar, B. A. Remington, and M. A. Meyers, "Void growth by dislocation emission," Acta Mater. 52(6), 1397-1408 (2004).

6J. R. Rice and R. Thomson, Philosophical Mag.: J. Theoretical Exp. Appl. Phys. 29, 73 (1974).

${ }^{7}$ V. V. Bulatov, W. G. Wolfer, and M. Kumar, "Shear impossibility: Comments on 'void growth by dislocation emission' and 'void growth in metals: Atomistic calculations'," Scr. Mater. 63(1), 144-147 (2010).

${ }^{8}$ J. W. Wilkerson and K. T. Ramesh, "A dynamic void growth model governed by dislocation kinetics," J. Mech. Phys. Solids 70, 262-280 (2014).

${ }^{9}$ P. Shanthraj and M. A. Zikry, "Dislocation-density mechanisms for void interactions in crystalline materials," Int. J. Plast. 34, 154-163 (2012).

${ }^{10}$ W. H. Liu, X. M. Zhang, J. G. Tang, and Y. X. Du, "Simulation of void growth and coalescence behavior with 3D crystal plasticity theory," Comput. Mater. Sci. 40(1), 130-139 (2007).

${ }^{11} \mathrm{~T}$. Ohashi, "Crystal plasticity analysis of dislocation emission from micro voids,” Int. J. Plast. 21(11), 2071-2088 (2005).

${ }^{12}$ G. P. Potirniche, J. L. Hearndon, M. F. Horstemeyer, and X. W. Ling, "Lattice orientation effects on void growth and coalescence in FCC single crystals," Int. J. Plast. 22(5), 921-942 (2006).

${ }^{13} \mathrm{M}$. Huang, Z. Li, and C. Wang, "Discrete dislocation dynamics modelling of microvoid growth and its intrinsic mechanism in single crystals," Acta Mater. 55(4), 1387-1396 (2007).

${ }^{14}$ H.-J. Chang, J. Segurado, and J. Llorca, "Three-dimensional dislocation dynamics analysis of size effects on void growth," Scr. Mater. 95, 11-14 (2015).

${ }^{15} \mathrm{~J}$. Segurado and J. Llorca, "Discrete dislocation dynamics analysis of the effect of lattice orientation on void growth in single crystals," Int. J. Plast. 26(6), 806-819 (2010).

${ }^{16}$ J. C. Crone, L. B. Munday, and J. Knap, "Capturing the effects of free surfaces on void strengthening with dislocation dynamics," Acta Mater. 101, 40-47 (2015).

${ }^{17} \mathrm{~T}$. Hatano and H. Matsui, "Molecular dynamics investigation of dislocation pinning by a nanovoid in copper," Phys. Rev. B 72(9), 094105 (2005).

${ }^{18} \mathrm{~T}$. Hatano, "Dislocation nucleation in shocked FCC solids: Effects of temperature and preexisting voids,” Phys. Rev. Lett. 93(8), 085501 (2004).

${ }^{19}$ S. Traiviratana, E. M. Bringa, D. J. Benson, and M. A. Meyers, "Void growth in metals: Atomistic calculations," Acta Mater. 56(15), 3874-3886 (2008).

${ }^{20}$ K. J. Zhao, C. Q. Chen, Y. P. Shen, and T. J. Lu, "Molecular dynamics study on the nano-void growth in face-centered cubic single crystal copper," Comput. Mater. Sci. 46(3), 749-754 (2009).

${ }^{21}$ Y. Tang, E. M. Bringa, and M. A. Meyers, "Ductile tensile failure in metals through initiation and growth of nanosized voids," Acta Mater. 60(12), 4856-4865 (2012).

${ }^{22}$ S. Rawat, M. Warrier, S. Chaturvedi, and V. M. Chavan, "Temperature sensitivity of void nucleation and growth parameters for single crystal copper: A molecular dynamics study,” Modell. Simul. Mater. Sci. Eng. 19(2), 025007 (2011).

${ }^{23}$ Y. Tang, E. M. Bringa, B. A. Remington, and M. A. Meyers, "Growth and collapse of nanovoids in tantalum monocrystals," Acta Mater. 59(4), 1354-1372 (2011)

${ }^{24} \mathrm{~S}$. Xu, Y. Su, D. Chen, and L. Li, "Plastic deformation of Cu single crystals containing an elliptic cylindrical void," Mater. Lett. 193, 283-287 (2017).

${ }^{25}$ C. Qiao, Y. Guo, Z. Wang, Y. Zheng, R. Zhang, L. Chen, Y.-L. Chen, W.-S. Su, Y. Jia, and S. Wang, "Effect of body defect on mechanical behaviors of $\mathrm{Cu}$ nanowire under tension: A molecular dynamics investigation,” J. Mater. Sci. 52(22), 13237-13246 (2017).

${ }^{26}$ C. Mi, D. A. Buttry, P. Sharma, and D. A. Kouris, "Atomistic insights into dislocation-based mechanisms of void growth and coalescence," J. Mech. Phys. Solids 59(9), 1858-1871 (2011).

${ }^{27}$ A. Montagne, V. Audurier, and C. Tromas, "Influence of pre-existing dislocations on the pop-in phenomenon during nanoindentation in $\mathrm{MgO}$," Acta Mater. 61(13), 4778-4786 (2013). 
${ }^{28}$ M. Damadam, S. Shao, I. Salehinia, I. Mastorakos, G. Ayoub, and H. M. Zbib, "Strength and plastic deformation behavior of nanolaminate composites with pre-existing dislocations," Comput. Mater. Sci. 138, 42-48 (2017).

${ }^{29}$ Q. Dong, Z. Yao, P. Saidi, and M. R. Daymond, "Effect of pre-existing dislocations on the formation of dislocation loops: Pure magnesium under electron irradiation," J. Nucl. Mater. 511, 43-55 (2018).

${ }^{30}$ D. C. Ahn, P. Sofronis, M. Kumar, J. Belak, and R. Minich, "Void growth by dislocation-loop emission," J. Appl. Phys. 101(6), 063514 (2007).

${ }^{31}$ S. Plimpton, "Fast parallel algorithms for short-range molecular dynamics," J. Comput. Phys. 117(1), 1-19 (1995).

${ }^{32}$ Y. Mishin, M. J. Mehl, D. A. Papaconstantopoulos, A. F. Voter, and J. D. Kress, "Structural stability and lattice defects in copper: Ab initio, tightbinding, and embedded-atom calculations," Phys. Rev. B 63(22), 224106 (2001).

${ }^{33}$ B. Cao, E. M. Bringa, and M. A. Meyers, "Shock compression of monocrystalline copper: Atomistic simulations," Metall. Mater. Trans. A 38(11), 2681-2688 (2007).

${ }^{34}$ E. Q. Lin, H. J. Shi, L. S. Niu, and E. Z. Jin, "Shock response of copper bicrystals with a (3 asymmetric tilt grain boundary," Comput. Mater. Sci. 59, 94-100 (2012).

${ }^{35}$ B. Li, L. Wang, H. H. Ma, J. C. E, and S. N. Luo, "Shock response of He bubbles in single crystal Cu," J. Appl. Phys. 116(21), 213506 (2014).

${ }^{36}$ P. Wen, G. Tao, C. Pang, S. Yuan, and Q. Wang, "A molecular dynamics study of the shock-induced defect microstructure in single crystal $\mathrm{Cu}$," Comput. Mater. Sci. 124, 304-310 (2016).

${ }^{37} \mathrm{~F}$. Yuan and $\mathrm{X}$. $\mathrm{Wu}$, "Hydrostatic pressure effects on deformation mechanisms of nanocrystalline FCC metals," Comput. Mater. Sci. 85, 8-15 (2014).

${ }^{38} \mathrm{M}$. A. Tschopp and D. L. McDowell, "Tension-compression asymmetry in homogeneous dislocation nucleation in single crystal copper," Appl. Phys. Lett. 90(12), 121916 (2007)

${ }^{39}$ M. A. Tschopp, D. E. Spearot, and D. L. McDowell, "Atomistic simulations of homogeneous dislocation nucleation in single crystal copper," Modell. Simul. Mater. Sci. Eng. 15(7), 693-709 (2007).

${ }^{40} \mathrm{Q}$. An, S.-N. Luo, L.-B. Han, L. Zheng, and O. Tschauner, "Melting of $\mathrm{Cu}$ under hydrostatic and shock wave loading to high pressures," J. Phys. Condens. Matter 20(9), 095220 (2008).

${ }^{41}$ W. Shinoda, M. Shiga, and M. Mikami, "Rapid estimation of elastic constants by molecular dynamics simulation under constant stress," Phys. Rev. B 69(13), 134103 (2004).

${ }^{42}$ C. D. Barrett, H. El Kadiri, and M. A. Tschopp, "Breakdown of the Schmid law in homogeneous and heterogeneous nucleation events of slip and twinning in magnesium," J. Mech. Phys. Solids 60(12), 2084-2099 (2012).

${ }^{43}$ M. P. Allen and D. J. Tildesley, Computer Simulation of Liquids (Clarendon Press, New York, 1989).
${ }^{44} \mathrm{~K}$. S. Cheung and S. Yip, "Atomic-level stress in an inhomogeneous system," J. Appl. Phys. 70(10), 5688-5690 (1991).

${ }^{45} \mathrm{~N}$. Chandra, S. Namilae, and C. Shet, "Local elastic properties of carbon nanotubes in the presence of Stone-Wales defects," Phys. Rev. B 69(9), 094101 (2004).

${ }^{46} \mathrm{~A}$. Stukowski and K. Albe, "Extracting dislocations and non-dislocation crystal defects from atomistic simulation data," Modell. Simul. Mater. Sci. Eng. 18(8), 085001 (2010)

${ }^{47}$ A. Stukowski, "Visualization and analysis of atomistic simulation data with OVITO-The open visualization tool," Modell. Simul. Mater. Sci. Eng. 18(1), 015012 (2010).

${ }^{48} \mathrm{~J}$. Hu, Z. Liu, K. Chen, and Z. Zhuang, "Investigations of shock-induced deformation and dislocation mechanism by a multiscale discrete dislocation plasticity model," Comput. Mater. Sci. 131, 78-85 (2017).

${ }^{49}$ R. A. Austin and D. L. McDowell, "Parameterization of a rate-dependent model of shock-induced plasticity for copper, nickel, and aluminum," Int. J. Plast. 32-33, 134-154 (2012).

${ }^{50}$ T. Tang, S. Kim, and M. F. Horstemeyer, "Molecular dynamics simulations of void growth and coalescence in single crystal magnesium," Acta Mater. 58(14), 4742-4759 (2010).

${ }^{51}$ J. P. Wang, J. W. Liang, Z. X. Wen, and Z. F. Yue, "Atomic simulation of void location effect on the void growth in nickel-based single crystal," Comput. Mater. Sci. 160, 245-255 (2019).

${ }^{52}$ G. I. Kanel, S. V. Razorenov, A. Bogatch, A. V. Utkin, V. E. Fortov, and D. E. Grady, "Spall fracture properties of aluminum and magnesium at high temperatures," J. Appl. Phys. 79(11), 8310-8317 (1996).

${ }^{53}$ E. Moshe, S. Eliezer, Z. Henis, M. Werdiger, E. Dekel, Y. Horovitz, S. Maman, I. B. Goldberg, and D. Eliezer, "Experimental measurements of the strength of metals approaching the theoretical limit predicted by the equation of state," Appl. Phys. Lett. 76(12), 1555-1557 (2000).

${ }^{54}$ J. Besson, "Damage of ductile materials deforming under multiple plastic or viscoplastic mechanisms," Int. J. Plast. 25(11), 2204-2221 (2009).

${ }^{55}$ S. J. A. Koh, H. P. Lee, C. Lu, and Q. H. Cheng, "Molecular dynamics simulation of a solid platinum nanowire under uniaxial tensile strain: Temperature and strain-rate effects," Phys. Rev. B 72(8), 085414 (2005).

${ }^{56}$ H. Ikeda, Y. Qi, T. Cagin, K. Samwer, W. L. Johnson, and W. A. Goddard, "Strain rate induced amorphization in metallic nanowires," Phys. Rev. Lett. 82(15), 2900-2903 (1999).

${ }^{57}$ A. S. J. Koh and H.-P. Lee, "Shock-induced localized amorphization in metallic nanorods with strain-rate-dependent characteristics," Nano Lett. 6(10), 2260-2267 (2006).

${ }^{58} \mathrm{H}$. Xie, F. Yin, T. Yu, G. Lu, and Y. Zhang, "A new strain-rate-induced deformation mechanism of $\mathrm{Cu}$ nanowire: Transition from dislocation nucleation to phase transformation," Acta Mater. 85, 191-198 (2015). 